\title{
Parcerias para o desenvolvimento produtivo (PDPs) como estratégia de acesso aos medicamentos ${ }^{1}$
}

\section{Partnerships for the productive development as strategy to medicine access}

\author{
${ }^{2}$ Bianca Bastos Macedo, ${ }^{3}$ Ana Claudia Dias de Oliveira \\ ${ }^{1}$ Monografia apresentada junto ao Curso de Pós-Graduação Lato Sensu de Especialização em Gestão da Inovação em Fitomedicamentos, \\ do Instituto de Tecnologia de Fármacos - Farmanguinhos / FIOCRUZ. \\ 2Instituto Vital Brazil. \\ ${ }^{3}$ Especialista Visitante do Centro de Desenvolvimento Tecnológico em Saúde da FIOCRUZ. \\ Correspondência: biancamacedo504@gmail.com
}

\section{Resumo}

Este trabalho apresentou uma abordagem teórica sobre as Parcerias para o Desenvolvimento Produtivo (PDPs) aplicado à área da saúde que visam à produção de medicamentos estratégicos para o Sistema Único de Saúde (SUS), cujas diretrizes estão previstas na Portaria do Ministério da Saúde GM/MS no 837, de 18 de abril de 2012. Essas parcerias são constituídas por laboratórios públicos nacionais e laboratórios privados, que a partir da Lista de Medicamentos Estratégicos disponibilizada pelo Ministério da Saúde, promovem o fortalecimento do complexo produtivo nacional bem como o desenvolvimento econômico, científico e tecnológico do país. Assim, sendo as PDPs consideradas instrumentos de políticas públicas, essas poderão ser utilizadas para apoiar a implementação da Política Nacional de Plantas Medicinais e Fitoterápicos, uma vez que a cooperação, a interação entre os atores envolvidos nas parcerias, poderá proporcionar o uso racional e sustentável da biodiversidade brasileira.

Palavras-chave: políticas públicas; saúde; preparações farmacêuticas.

\begin{abstract}
This paper presents a theoretical approach about the Partnerships for the Productive Development applied to healthcare aimed at the production of strategic medicines for the Sistema Único de Saúde (SUS), whose guidelines are laid down by Ministério da Saúde ordinance GM / MS No. 837 of 18 April 2012. These partnerships are made up of national public laboratories and private laboratories, which from the List of Strategic Drug available by the Ministério da Saúde, promote the strengthening of national production complex as well as economic development, science and technology in the country. Thus, the Partnerships for the Productive Development
\end{abstract}


being considered public policy instruments, these can be used to support the implementation of the Política Nacional de Plantas Medicinais e Fitoterápicos, since cooperation, interaction between the actors involved in the partnerships, can provide the rational and sustainable use Brazilian biodiversity.

Keywords: public policies; health; pharmaceutical preparations.

\section{Introdução}

O Sistema Único de Saúde (SUS) conforme reza o artigo 6 da sua Lei Orgânica (Brasil, 1990) participa da produção de medicamentos, e ainda da formulação da política de medicamentos. Nesse sentido, foi aprovada a Política Nacional de Medicamentos (PNM) que visa garantir à população brasileira o acesso a medicamentos considerados essenciais, seguros, eficazes e de qualidade, bem como o uso racional (Brasil, 1998).

Assim, de forma a melhorar a promoção do acesso igualitário e universal às ações e serviços de saúde, o Brasil redefiniu as suas estratégias de política industrial e priorizaram alguns setores, que no presente estudo está relacionado ao setor farmacêutico, mediante o fortalecimento do Complexo Econômico Industrial da Saúde, o incentivo à capacitação tecnológica dos produtores nacionais públicos e privados, e o estímulo à inovação com investimentos em pesquisa e desenvolvimento.

Esta nova perspectiva política industrial na área da saúde acarretou na utilização das Parcerias de Desenvolvimento Produtivo (PDP), estabelecidas pela Portaria GM/MS № 837 (Brasil, 2012), do Ministério da Saúde.

As parcerias em comento têm como objetivos: (a) racionalização do poder de compra do Estado, mediante a centralização seletiva dos gastos na área da saúde, com vistas à diminuição nos custos de aquisição do SUS e à viabilização da produção no País de produtos inovadores de alta essencialidade para a saúde, tendo como foco a melhoria do acesso da população a insumos estratégicos; (b) fomento ao desenvolvimento tecnológico conjunto e ao intercâmbio de conhecimentos para a inovação no âmbito dos produtores públicos e privados nacionais, tornando-os competitivos e capacitados a enfrentar a concorrência global em bases permanentes num contexto de incessante mudança tecnológica; (c) foco na fabricação local de produtos estratégicos para o SUS e de produtos de alto custo e/ou de grande impacto sanitário e social, aliando o pleno abastecimento e diminuição da vulnerabilidade do SUS com a ampliação da riqueza do País; (d) negociação de reduções significativas e progressivas de preços na medida em que a tecnologia é transferida e desenvolvida, conforme seja considerada estratégica para o SUS (Brasil, 2012). Com isso, o presente estudo objetiva demonstrar os resultados sociais, econômicos e políticos gerados por essas parcerias como forma de aplicar este modelo das PDPs para os medicamentos fitoterápicos.

\section{Materiais e Métodos}

Foi realizada uma pesquisa descritiva com análise de conteúdo a partir de documentos e publicações oficiais disponíveis em sites oficiais, tais como ABIFINA, ALFOB, ANVISA, SCITE, PROGENÉRICOS, e análise de artigos jurídicos. 


\section{Resultados e Discussão}

Observa-se na FIGURA 1 uma linha do tempo a respeito da evolução das políticas públicas no setor farmacêutico.

As PDPs têm como diretrizes proporcionar o acesso às tecnologias prioritárias, reduzir os preços dos medicamentos e produtos para saúde, internalizar e desenvolver novas tecnologias de alto valor agregado e promover a articulação entre as instituições públicas e privadas.

O estudo apresentado demonstra valorização das parcerias de desenvolvimento produtivo como instrumentos de políticas públicas na área da saúde, cujo principal objetivo é o melhoramento do acesso da população a medicamentos considerados estratégicos para o SUS. O fortalecimento e a importância que foi conferida aos laboratórios públicos oficiais e as empresas privadas nacionais integrantes dessas alianças permitem que o setor produtivo farmacêutico no país cresça e se aperfeiçoe com tecnologias inovadoras e competitivas, capazes de gerar processos e produtos inovadores para a sociedade.

Em razão dos resultados expressivos e o grande número de parcerias celebradas, o Ministério da Saúde com o intuito de aprimorar ainda mais a regulamentação das PDPs, lançou em 2014 a Consulta Pública no 08/GM/MS (Brasil, 2014), relativa ao texto da minuta de Portaria que "redefine as diretrizes e os critérios para a definição da lista de produtos estratégicos para o Sistema Único de Saúde (SUS) e o estabelecimento das Parcerias para o Desenvolvimento Produtivo (PDPs) e disciplina os respectivos processos de submissão, instrução, decisão, transferência e absorção de tecnologia, aquisição de produtos estratégicos para o SUS no âmbito das PDPs, monitoramento e avaliação" (Brasil, 2014).

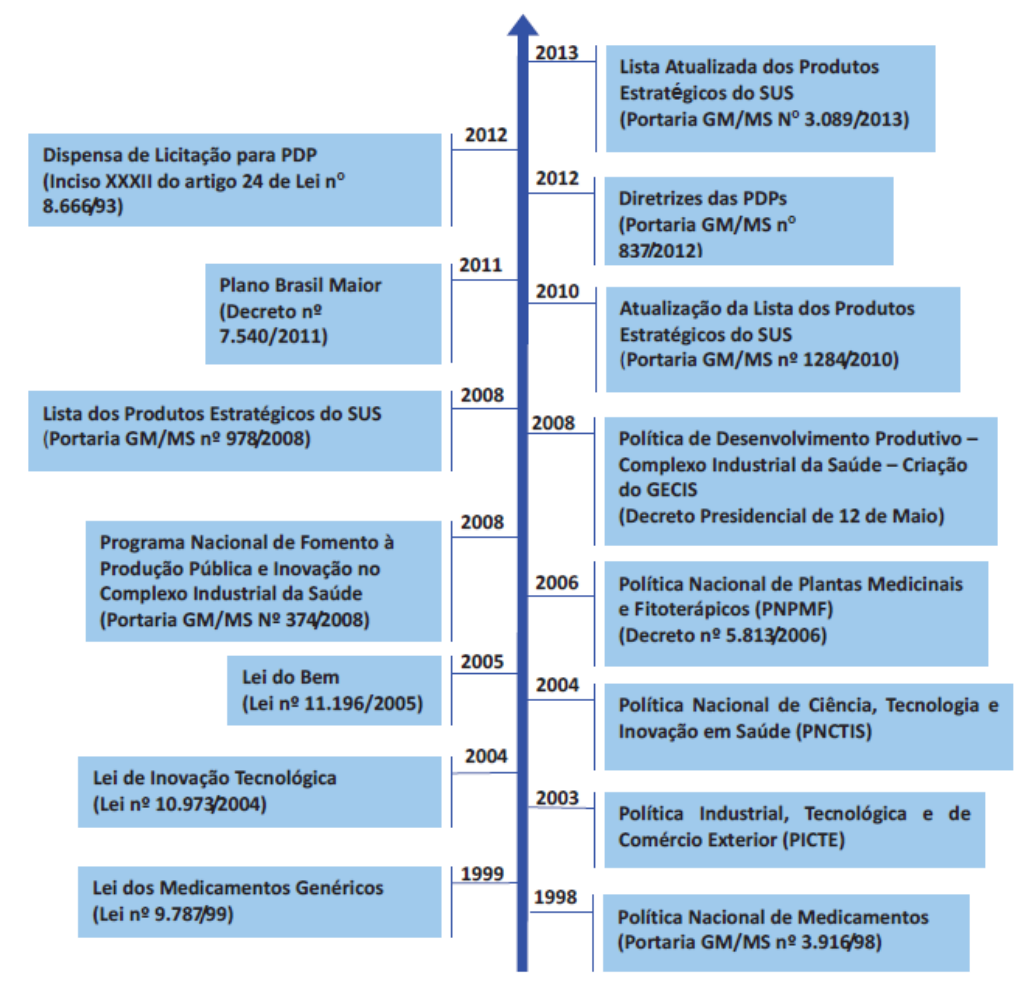

FIGURA 1. Evoluções das Políticas Públicas no Setor Farmacêutico Brasileiro. Fonte: Autor. 


\section{Conclusões}

Observa-se que o cenário se encontra propício para o estabelecimento deste modelo de parcerias no desenvolvimento da cadeia produtiva dos medicamentos fitoterápicos. O primeiro passo já foi dado com a inclusão dos fitoterápicos na Lista de Produtos Estratégicos do SUS, ademais os produtores públicos estão se equipando através de contratação de recursos humanos qualificados, a aquisição de novos equipamentos, reformas de laboratórios. $E$ os parceiros privados estão investindo cada vez mais em suas plantas industriais e em pesquisa e desenvolvimento. Nesse sentido, a PDP poderá ser usada como uma forma de estimular a promoção do uso sustentável da biodiversidade e fomentar a produção dos medicamentos fitoterápicos com segurança, eficácia e qualidade no SUS, de modo a torná-los mais acessíveis à população como pretende a Política Nacional de Plantas Medicinais e Fitoterápicos.

\section{Referências}

BRASIL. 1990. Congresso Nacional. Lei no 8080, de 19 de setembro de 1990. Dispõe sobre as condições para a promoção, proteção e recuperação da saúde, a organização e o funcionamento dos serviços correspondentes e dá outras providências. Brasília. DOU de 20/09/1990.

BRASIL. 1998. Ministério da Saúde. Portaria GM/MS n. 3.916, de 30 de outubro de 1998. Aprova a Política Nacional de Medicamentos. Brasília. DOU de 10/11/1998.

BRASIL. 2012. Ministério da Saúde. Portaria GM/MS no 837, de 18 de abril de 2012. Define as diretrizes e os critérios para o estabelecimento das Parcerias para o Desenvolvimento Produtivo (PDP). Brasília. DOU de 27/04/2012.

BRASIL. 2014. Ministério da Saúde. Consulta Pública GM/MS n0 08, de 13 de agosto de 2014. Redefine as diretrizes e os critérios para a definição da lista de produtos estratégicos para o Sistema Único de Saúde (SUS) e o estabelecimento das Parcerias para o Desenvolvimento Produtivo (PDP) e disciplina os respectivos processos de submissão, instrução, decisão, transferência e absorção de tecnologia, aquisição de produtos estratégicos para o SUS no âmbito das PDP, monitoramento e avaliação. Brasília. DOU de 14/08/2014. 